\title{
Cooperative Beamforming Research for Two-Way Relay in Cognitive Radio by a New SDP Method
}

\author{
Hongyan $\mathrm{Cai}^{1,2}$ and Wenbao $\mathrm{Ai}^{3}$ \\ ${ }^{1}$ School of Economics and Management, Beijing University of post and \\ Telecommunication, Beijing, China \\ ${ }^{2}$ School of Mathematics and Statistics, Hebei University of Economics and business, \\ Shijiazhuang, China \\ ${ }^{3}$ School of Sciences, Beijing University of post and telecommunication Beijing, China \\ hy_cai75@163.com,wenbaoai@gmail.com
}

\begin{abstract}
In this paper we investigate the problem of cooperative beamforming design in cognitive radio network with a primary network and a secondary network. In the secondary network, the two transmitters communicate by the help of relays each other, and the relays select the amplify-and-forward $(A F)$. The aim of this paper is to minimize the total power of relays in the secondary network with the SNRs of two transmitters under constraints and the QoS requirement of the primary user. Therefore, we design an optimization model with constrains. We consider a SDP relaxation method and prove that there is a rank-one optimal solution which is the optimal solution of the original problem. So we obtain weights of the beamforming and the optimal power of relays. Moreover, we advise a reasonable number of the relays by numerical simulation.
\end{abstract}

Keywords: Cognitive relay network, Cooperative beamforming, Optimization, SDP

\section{Introduction}

In this paper, we consider cognitive relay network (CNR), which includes both parts: the cognitive radio network and the relay network. We know that the cognitive radio network is an effective way in improving the spectrum utilization rate and in coping with the spectral limitations. Spectrum scarcity in wireless networks allocation becomes a major problem that has triggered a broad range of research activities because of fixed spectrum. The spectrum utilization efficiency of wireless systems by adopting the innovative idea of cognitive radio (CR), which allows the secondary users (SUs) to dynamically access the licensed spectrum originally allocated to the primary users (PUs) [1]. Cognitive users detect the given channel environment at specific times actively and change their transmission or reception parameters to communicate efficiently for avoiding interference with licensed or unlicensed users [2-3]. The major challenge for CR networks is to ensure the quality-of-service (QoS) of PUs while trying to maximize the throughput of SUs. When SUs are allowed to use the spectrum concurrently with a PU, the resultant interference power at the PU has to be kept below a certain threshold [3, 4] .This constraint limits the allowed transmit power of SUs and the throughput of SUs.

The relay network is a powerful technology, which can combat signal fading due to multipath propagation in a wireless medium. Cognitive relay network is a potential way to improve the performance of SUs and guarantee the Qos of PUs. In [5-7], the authors 
investigate the outage probability of SUs in the cognitive relay network. Cooperative beamforming which is used in cognitive radio network is another technology. It mitigates the interference power on the primary users and improves the secondary performance. A cognitive relay network includes an additional constraint corresponding to the interference effects on the primary users, which increase the complexity of the problem. The idea of using a single-antenna relay station (RS) to implement the one-way relaying in CR networks has attracted a great deal of attention lately [8]. The results obtained show the throughput improvement of SUs. We will adopt this idea in this paper. Cooperative beamforming which has been studied in the traditional relay network successfully is very new to the cognitive networks [8]

In this paper, we will study the advantage of choosing cooperative beamforming in CR networks. We consider an underlay CR network which includes a primary and a secondary network which is two- way transmission. The relay strategy selects the amplify-and-forward (AF) protocol for simplifying the system.

The rest of the paper is organized as follows. Section 2 provides the details of the system model. Section 3 gives the details of the signal model. Section 4 proposes the method of solving optimal problem. Section 5 and Section 6 provide the numerical simulations and conclusion respectively.

Throughout this paper, we adopt the following notations:

We define $E[x]$ as the expectation of, $x^{H}$ is the conjugate transpose of $x$, and $\operatorname{rank}(X)$ , trace $(X)$ represent the rank and the trace of matrix $X$ respectively.

\section{System Model}

We study that a cognitive radio network shares spectrum resources with a primary network. The cognitive radio network (CRN) as shown in Figure 1. It includes two parts as: primary network (PN) with the primary transmitter (PT) and the primary destination (PD), and secondary network $(\mathrm{SN})$ with the secondary transmitters $(\mathrm{S} 1, \mathrm{~S} 2), N$ relays $\left\{S R_{i}\right\}_{i=1}^{N}$. All nodes in network have been configured with one antenna. We assume that there is no direct link between the transmitters, the help of relays is necessary to establish the two-way communication link in the secondary network. We assume S1, S2 are far from PD and PT is far from S1 and S2, so the interferences between them may be ignored and we assume a flat fading scenario. We denote the channel between PT and PD as $h_{P D}, g_{P R_{i}} \in \square, i=1,2, \cdots, N$ denote the channel between PT and relays, $h_{S_{1} R_{i}}, h_{S_{2} R_{i}}, g_{R_{i} D} \in \square, i=1,2, \cdots, N$ are the channel between $\mathrm{S} 1$ and relays, between $\mathrm{S} 2$ and relays, between relays and PD respectively. 


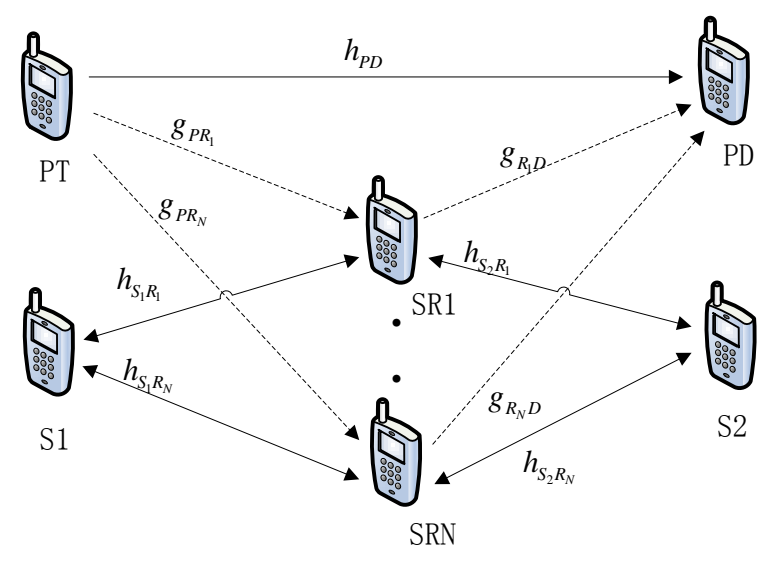

Figure 1. System Model

The two transmitters in the $\mathrm{CN}$ divide the process into two sub-processes. In the first sub-process, PT transmits message $s 1$ to $\mathrm{PD}, \mathrm{S} 1$ and $\mathrm{S} 2$ transmit message $x_{1}, x_{2}$ to the relays SR respectively. In the second sub-process, PT transmits message $s 2$ to PD and SRs retransmit the received signal to $\mathrm{S} 1$ and $\mathrm{S} 2$ respectively. We herein use a two-step amplify-and-forward (AF) protocol. To simplify the analysis, we assume that $x_{1}, x_{2}, s 1, s 2$ are independent to each other, and $E\left[\left|x_{1}\right|^{2}\right]=1, E\left[\left|x_{2}\right|^{2}\right]=1, E\left[|s 1|^{2}\right]=1, E\left[|s 2|^{2}\right]=1$.Our aim is to obtain the optimal beamforming weight coefficient and minimum transmission power of the relays in ensuring the QoS of the primary network users.

\section{Signal Model}

In this section, we will deduce the optimal signal model for the transmission power of relay beamforming. For the practical purpose, we assume the half-duplex transmission and the time-division duplex is employed.

We denote $P_{P}, P_{1}, P_{2}$ as the transmit power of PT, S1 and S2.

In the first sub-process:

The received signal at $i$ th relay is given as follows:

$$
r_{i}=\sqrt{P_{1}} h_{S_{1} R_{i}} x_{1}+\sqrt{P_{2}} h_{S_{2} R_{i}} x_{2}+\sqrt{P_{P}} g_{P R_{i}} s_{1}+n_{R_{i}}
$$

where $n_{R_{i}}: N\left(0, N_{R}\right)$ represents the complex Gaussian noise at the ${ }_{i}$ th relay.

In the second sub-process:

The received signals of $\mathrm{PD}, \mathrm{S} 1$ and $\mathrm{S} 2$ are respectively: 


$$
\begin{aligned}
& y_{D}=\sqrt{P_{P}} h_{P D} s_{2}+\stackrel{\circ}{\stackrel{N}{a}} g_{i=1} w_{i} w_{i} r_{i}+n_{D}
\end{aligned}
$$

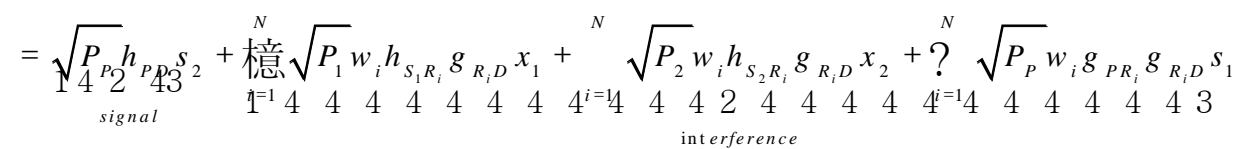

$$
\begin{aligned}
& +\stackrel{N}{\mathrm{a}} w_{i} g_{R_{i} D} n_{R_{i}}+n_{D} \\
& l^{1} 4 \begin{array}{llllll}
4 & 2 & 4 & 4 & 3
\end{array} \\
& y_{S 1}=\stackrel{\complement}{i=1}_{i=1}^{N} h_{S_{1} R_{i}} w_{i} r_{i}+n_{S_{1}} \\
& =\underset{i=1}{\text { 檍 }} \underset{P_{1}}{N} w_{i}\left|h_{S_{1} R_{i}}\right|^{2} x_{1}+{ }_{i=1}^{N} \sqrt{P_{2}} w_{i} h_{S_{1} R_{i}} h_{S_{2} R_{i}} x_{2}+\underset{\substack{\text { 檍 } \\
i=1}}{N} \sqrt{P_{P}} w_{i} g_{P R_{i}} h_{S_{1} R_{i}} s_{1}+{ }_{i=1}^{N} w_{i} h_{S_{1} R_{i}} n_{R_{i}}+n_{S_{1}}
\end{aligned}
$$

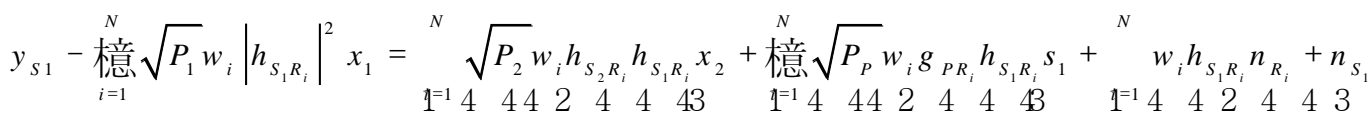

$$
\begin{aligned}
& y_{S 2}=\stackrel{\circ}{\stackrel{N}{a}} h_{S_{2} R_{i}} w_{i} r_{i}+n_{S_{2}}
\end{aligned}
$$

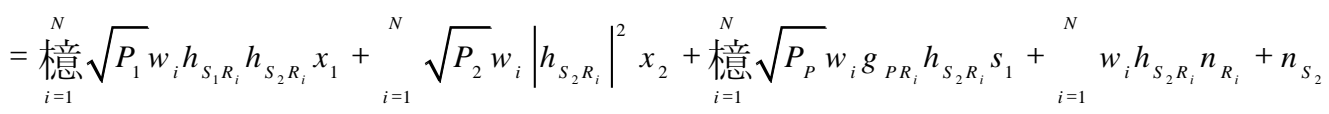

$$
\begin{aligned}
& y_{S_{2}}-\stackrel{\triangleright}{a}_{i=1}^{N} \sqrt{P_{2}} w_{i}\left|h_{S_{2} R_{i}}\right|^{2} x_{2} \\
& =\stackrel{N}{N} \text { 檍 } \sqrt{P_{1}} w_{i} h_{S_{1} R_{i}} h_{S_{2} R_{i}} x_{1}+{ }^{N} \sqrt{P_{P}} w_{i} g_{P R_{i}} h_{S_{2} R_{i}} s_{1}+?{ }^{N} w_{i} h_{S_{2} R_{i}} n_{R_{i}}+n_{S_{2}}
\end{aligned}
$$

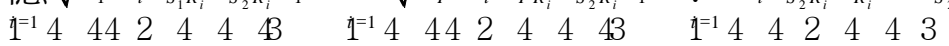

where, $n_{D} \square N(0,1), n_{S_{1}} \square N(0,1), n_{S_{2}} \square N(0,1)$ denote the complex Gaussian noise of PD,S1 and $\mathrm{S} 2$, and $w_{i} \in \quad$ represent the beamforming weight of the $i$ relay.

The disturbed power of PD is

$$
\begin{aligned}
& I=P_{1}\left|\begin{array}{|l}
\left.\right|_{i=1} ^{N} \\
\text { 檍 } \\
i=1
\end{array} h_{S_{1} R_{i}} g_{R_{i} D}\right|^{2}+P_{2}\left|{ }_{i=1}^{N} w_{i} h_{S_{2} R_{i}} g_{R_{i} D}\right|^{2}+P_{P}\left|?_{i=1}^{N} w_{i} g_{P R_{i}} g_{R_{i} D}\right|^{2} \\
& =P_{1} w^{H} \stackrel{\circ}{h_{1}} \stackrel{\circ}{h}_{1}^{H} w+P_{2} w^{H} \stackrel{\circ}{h_{2}} \stackrel{\circ}{h}_{2}^{H} w+P_{P} w^{H} g g^{H} w
\end{aligned}
$$

The SNR of $\mathrm{S} 1$ is

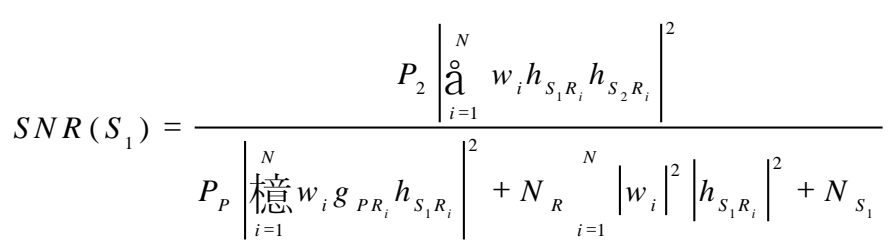




$$
=\frac{P_{2} w^{H} h h^{H} w}{P_{P} w{ }^{H} \stackrel{\circ}{g}_{1} \stackrel{\circ}{g}_{1}^{H} w+N_{R} w^{H} H_{1} H_{1}^{H} w+N_{S_{1}}}
$$

The SNR of $\mathrm{S} 2$ is

$$
\begin{aligned}
& \operatorname{SNR}\left(S_{2}\right)=\frac{\left.P_{1}||_{i=1}^{N} w_{i} h_{S_{1} R_{i}} h_{S_{2} R_{i}}\right|^{2}}{\left.\left.P_{P}\right|_{\substack{\text { 檍 } \\
i=1}} ^{N} w_{i} g_{P R_{i}} h_{S_{2} R_{i}}\right|^{2}+N_{R}{ }_{i=1}^{N}\left|w_{i}\right|^{2}\left|h_{S_{2} R_{i}}\right|^{2}+N_{S_{2}}} \\
& =\frac{P_{1} w^{H} h h^{H} w}{P_{P} w{ }^{H} \stackrel{\circ}{g}_{2} \stackrel{\circ}{g}_{2}^{H} w+N_{R} w^{H} H_{2} H_{2}{ }_{2}^{H} w+N_{S_{2}}}
\end{aligned}
$$

The transmitted signal of the $i$ relay is $w_{i} r_{i}$, the transmitted power is

$$
\left|w_{i}\right|^{2}\left(P_{1}\left|h_{S_{1} R_{i}}\right|^{2}+P_{2}\left|h_{S_{1} R_{i}}\right|^{2}+P_{P}\left|g_{P R_{i}}\right|^{2}+N_{R}\right),
$$

The total power of relays:

$$
\begin{aligned}
P_{R} & =\stackrel{\mathrm{a}}{i=1}_{i=1}^{N}\left|w_{i}\right|^{2}\left(P_{1}\left|h_{S_{1} R_{i}}\right|^{2}+P_{2}\left|h_{S_{1} R_{i}}\right|^{2}+P_{P}\left|g_{P R_{i}}\right|^{2}+N_{R}\right) \\
& =P_{1} w^{H} H_{1} H_{1}{ }^{H} w+P_{2} w^{H} H_{2} H_{2}{ }^{H} w+P_{P} w^{H} G_{1} G_{1}{ }^{H} w+N_{R} w^{H} w
\end{aligned}
$$

Where,

$$
\begin{array}{ll}
w=\left[w_{1}, w_{2}, \ldots, w_{N}\right]^{H}, h=\left[h_{S_{1} R_{1}} h_{S_{2} R_{1}}, h_{S_{1} R_{2}} h_{S_{2} R_{2}}, \ldots, h_{S_{1} R_{N}} h_{S_{2} R_{N}}\right]^{H}, \\
h_{1}=\left[h_{S_{1} R_{1}} g_{R_{1} D}, h_{S_{1} R_{2}} g_{R_{2} D}, \ldots, h_{S_{1} R_{N}} g_{R_{N} D}\right]^{H}, & h_{2}=\left[h_{S_{2} R_{1}} g_{R D_{1}}, h_{S R_{2}} g_{R_{2} D}, . h_{S R_{N}} g_{2^{R_{N}} D}{ }^{H},\right. \\
g=\left[g_{P R_{1}} g_{R_{1} D}, g_{P R_{2}} g_{R_{2} D}, \ldots, g_{P R_{N}} g_{R_{N} D}\right]^{H}, & g_{1}=\left[g_{P R_{1}} h_{S_{1} R_{1}}, g_{P R_{2}} h_{S_{1} R_{2}}, \ldots, g_{P R_{N}} h_{S_{1} R_{N}}\right]^{H}, \\
g_{2}=\left[g_{P R_{1}} h_{S_{2} R_{1}}, g_{P R_{2}} h_{S_{2} R_{2}}, \ldots, g_{P R_{N}} h_{S_{2} R_{N}}\right]^{H}, & H_{1}=\operatorname{diag}\left[h_{S_{1} R_{1}}, h_{S_{1} R_{2}}, \ldots, h_{S_{1} R_{N}}\right], \\
H_{2}=\operatorname{diag}\left[h_{S_{2} R_{1}}, h_{S_{2} R_{2}}, \ldots, h_{S_{2} R_{N}}\right], & G_{1}=\operatorname{diag}\left[g_{P R_{1}}, g_{P R_{2}}, \ldots, g_{P R_{N}}\right], \\
G_{2}=\operatorname{diag}\left[g_{R_{1} D}, g_{R_{2} D}, \ldots, g_{R_{N} D}\right] . &
\end{array}
$$

\section{The Total Power of Relays}

In the section, our aim is to obtain the optimal beamforming weight coefficient and minimum transmission power of the relays. But we must ensure the GoS of the primary network users, which is that the interference is less than the threshold $\eta$ given in advance for primary network users, moreover, we must ensure that the SNRs of the secondary network users are larger than some threshold $\gamma_{1}, \gamma_{2}$.

So, the optimal model can write as following: 
International Journal of Future Generation Communication and Networking

$$
\begin{aligned}
& f^{*}=\min _{w} \quad P_{1}\left(1+w^{H} H_{1} H_{1}^{H} w\right)+P_{2}\left(1+w^{H} H_{2} H_{2}{ }^{H} w\right)+w^{H}\left(P_{P} G_{1} G_{1}{ }^{H}+N_{R} I\right) w \\
& \text { s.t. } \frac{P_{2} w^{H} h h^{H} w}{w^{H}\left(P_{P} \stackrel{\circ}{g_{1}} \stackrel{\circ}{g_{1}^{H}}+N_{R} H_{1} H_{1}^{H}\right) w+N_{S_{1}}} g_{1} \\
& \frac{P_{1} w^{H} h h^{H} w}{w^{H}\left(P_{P} \stackrel{\circ}{g}_{2}^{\circ} \stackrel{\circ}{H}_{2}^{H}+N_{R} H_{2} H_{2}^{H}\right) w+N_{S_{2}}} g_{2} \\
& P_{1} w^{H} \stackrel{\circ}{h} \stackrel{\circ}{h}_{1}^{H} w+P_{2} w^{H} \stackrel{\circ}{h} \stackrel{\circ}{h}_{2}^{H} w+P_{P} w^{H} g g^{H} w ? h
\end{aligned}
$$

(11) is equivalent as following:

$$
\begin{gathered}
f \not^{*}=f^{*}-P_{1}-P_{2}=\underset{w}{\min } w^{H} A w \\
\text { s.t. } \quad w^{H} B_{1} w \quad{ }^{3} 1, \\
w^{H} B_{2} w^{3} 1, \\
w^{H} C w £ 1 .
\end{gathered}
$$

Where, $A=A_{1}+A_{2}+A_{3}, A_{1}=H_{1} H_{1}{ }^{H}, A_{2}=H_{2} H_{2}{ }_{2}^{H}, A_{3}=P_{P} G_{1} G_{1}{ }^{H}+N_{R} I$,

$$
\begin{aligned}
& B_{1}=\left[P_{2} h h^{H}-g_{1}\left(P_{P} \stackrel{\circ}{g_{1}} \stackrel{\circ}{g}_{1}^{H}+N_{R} H_{1} H_{1}^{H}\right)\right] / g_{1} N_{S_{1}}, \\
& B_{2}=\left[P_{1} h h^{H}-g_{2}\left(P_{P} \stackrel{\circ}{g}_{2} \stackrel{\circ}{g}_{2}^{H}+N_{R} H_{2} H_{2}^{H}\right)\right] / g_{2} N_{S_{2}}, C=\left[P_{1} \stackrel{\circ}{h}_{1}^{\circ} \stackrel{\circ}{h}^{H}+P_{2} \stackrel{\circ}{h}_{2}^{\circ} \stackrel{H}{h}^{H}+P_{P} g g^{H}\right] / h .
\end{aligned}
$$

Let $W=w w^{H}$, rewrite the optimization problem

$$
\begin{aligned}
& f \not \not^{*}=\mathrm{min} \quad W \mathrm{gA} \\
& \text { s.t. } \quad B_{1} \mathrm{~g} W \quad \text { 납 } 1, B_{2} \mathrm{~g} W \quad 1, \\
& \quad C \mathrm{~g} W ? 1, \operatorname{Rank}(W) \quad 1, \quad W \text { f } 0 .
\end{aligned}
$$

The SDP relaxation of (13) is

$$
\begin{aligned}
& \mathrm{m} \text { in } \quad W \mathrm{~g} A \\
& \text { s.t. } \quad B_{1} \mathrm{gW} \quad \text { 납1, } B_{2} \mathrm{~g} W \quad 1 \\
& C \mathrm{~g} W £ 1, \quad W \mathrm{f} 0 \text {. }
\end{aligned}
$$

The dual problem is

The KKT conditions are:

$$
\begin{array}{ll} 
& \max y_{1}+y_{2}+y_{3} \\
\text { s.t. } & y_{1} B_{1}+y_{2} B_{2}+y_{3} C+S=A, \\
& y_{1}, y_{2} \text { 납 } 0, y_{3} \quad 0, S \text { f } 0
\end{array}
$$

$$
\begin{gathered}
S^{*}=A-y_{1}^{*} B_{1}-y_{2}^{*} B_{2}-y_{3} C \underline{\mathrm{f}} 0 \\
y_{1}^{*}\left(1-B_{1} \mathrm{~g} W^{*}\right)=0 \\
y_{2}^{*}\left(1-B_{2} \mathrm{~g} W{ }^{*}\right)=0 \\
y_{3}^{*}\left(1-C \mathrm{~g} W{ }^{*}\right)=0 \\
S^{*} \mathrm{~g} W^{*}=0
\end{gathered}
$$


$y_{1}^{*}$ 납 $0, y_{2}^{*} \quad 0, y_{3}^{*} ? 0$ is the feasible solution of (15), and $W^{*}$ is the minimum matrix solution.

Theorem For the optimum $W^{*}$ of (14), one can find in polynomial-time a nonzero vector $w^{*} \hat{\mathrm{I}} £^{N}$, such that $W^{\% 0^{*}}=w^{*} w^{* H}$ is the optimal solution of (13) by rank- one decomposition of $W^{*}$, therefore $w^{*}$ is the optimal solution of (11).

Proof if $\operatorname{rank}\left(W^{*}\right)=1, W^{*}=w^{*} w^{* H}$ is the optimal solution of (13). Otherwise ,if $\operatorname{rank}\left(W^{*}\right)=r ? 2$, we consider this problem in three cases:

(1) When $y_{1}^{*}{ }^{1} \quad 0, y_{2}^{*}=0, y_{3}^{*}=0$,

(2) When $y_{3}{ }^{*}=0, y_{2}{ }^{*}{ }^{1} 0, y_{1}^{*}{ }^{*} \quad 0$,

Please see the theorem 4.2.2 of the reference [9].

(3) When $y_{3}{ }^{*}{ }^{1} 0, y_{2}^{*}{ }^{1} \quad 0, y_{1}^{*}{ }^{*} 0$,

From the theorem 2.1 of [10] and [11], there must be a rank-one decomposition of $W^{*}$, that is

$$
\begin{aligned}
& W^{*}=\stackrel{\mathrm{a}}{r}_{j=1}^{r} w_{j}^{*} w_{j}^{* H} \text { such that } \\
& w_{j}{ }^{* H}\left(B_{1}-B_{2}\right) w_{j}{ }_{j}^{*}=\left(B_{1}-B_{2}\right) ? w_{j}{ }^{*} w_{j}{ }_{j} H \quad \frac{\left(B_{1}-B_{2}\right) ? W^{*}}{r}=0 \\
& w_{j}^{* H}\left(B_{1}-C\right) w_{j}^{*}=\left(B_{1}-C\right) ? w_{j}^{*} w_{j}^{* H} \quad \frac{\left(B_{1}-C\right) ? W^{*}}{r}=0, j=1, \mathrm{~L}, r .
\end{aligned}
$$

Because of $C \mathrm{~g} W^{*}=C \underset{j=1}{\stackrel{r}{r}} w_{j} w_{j}^{H}=1$, there must be a $j_{0}$, such that $C \mathrm{~g} w_{j_{0}} w_{j_{0}}^{H}>0$, let $w^{*}=\frac{1}{\sqrt{C g w_{j_{0}} w_{j_{0}}^{H}}} w_{j_{0}}, W^{Q / 0^{*}}=w^{*} w^{* H}$.

Because of $S^{*} \mathrm{~g} W{ }^{*}=0, \stackrel{\mathrm{a}}{j=1}_{j}^{r} w_{j}^{* H} S^{*} w_{j}^{*}=0$, and since $S^{*} \underline{\underline{f}} 0$, so $S^{*} \mathrm{~g} w_{j}^{* H} w_{j}^{*}=0, j=1,2, \mathrm{~L}, r, \mathrm{SO} \quad S^{*} \mathrm{gw}^{*} w^{* H}=0$.

$C \mathrm{~g} W^{*} 0^{*}=C \frac{1}{C g w_{j_{0}} w_{j_{0}}^{H}} w_{j_{0}} w_{j_{0}}^{H}=1,\left(B_{1}-B_{2}\right) ? w w^{H} \quad \frac{\left(B_{1}-B_{2}\right) ? w_{j_{0}} w_{j_{0}}^{H}}{C g w_{j_{0}} w_{j_{0}}^{H}}=0$, $\left(B_{1}-C\right) ? w w^{H} \quad \frac{\left(B_{1}-C\right) ? w_{j_{0}} w_{j_{0}}{ }^{H}}{C g w_{j_{0}} w_{j_{0}}^{H}}=0$, so there is $B_{1} ? w w^{H} \quad B_{2} ? w w^{H} \quad C$ g $w w^{H}=1$.

So, the algorithm for solving the optimal problem (11) in order to obtain the optimal beamforming weights and the minimal power of relays is summarized below: 


\title{
Table 1. Iteration Algorithm for the SDP Program
}

\author{
1. initialization $P_{1}, P_{2}, P T, d$ \\ 2. repeat \\ 3. $k=k+1$ \\ 4. solve SDP problem to get the optimal value $f^{*}$ and the optimal matrix $W^{*}$ \\ 5. obtain $w^{*}$ based on the proof of the Theorem \\ 6. output $f^{*}$ and $w^{*}$
}

\section{Numerical Simulations}

In this section, we do many simulations to study the performance of the secondary network under different situations, and these simulations are based on independent Rayleigh flat-fading channels. The relays are uniformly distributed over the region whose center is at $(0$, 0 ) and radius is 0.8 . PT, PD, S1, and S2 are at $(-0.75,1.5),(0.75,1.5),(-1,0)$ and $(1,0)$ respectively. The noise of S1 and S2 is 1[12-13]. We design the optimal model that minimize the total transmitter power of relays. We discuss the cases of $\mathrm{N}=10,15,20$. And let $P_{1}=P_{2}=P T=15 d B, d=5 d B$. Fig 2 depicts that the total transmitter power of relays become large with the increase of SNRs of secondary network regardless of how many the relay number is. When SNRs $>12 \mathrm{Db}$, the total transmitter power of relays rise rapidly with the increase of SNRs. Simultaneously, we can see that the total transmitter power of relays decreases significantly with the increase of the relay number. Moreover, when SNRs are larger requested, the decrease of the total transmitter power is very obvious. This conclusion is described to increase the number of relay the relay can decrease the total transmit power. However, since the increased number of relays is bound to be affected by cost constraints, we think the relays number 20 is more reasonable, which is because the minimum transmit power of the relays are relatively close in 15 and 20. It is unworthy to get such little gain to use so more relays in practice.

\section{Conclusion}

In this paper, we study a cooperative and cognitive radio system where a cognitive RS aids two SUs in exchanging their information while maintaining the interference power at a PU under a certain threshold. Two-way relaying is employed in order to obtain high spectrum efficiency. We provide the structure of the optimal relay BF that minimizes the achievable powers of relay. We design an algorithm based on the SDP relaxation method for this model and prove that there is a rank-one optimal solution which is the optimal solution of the original problem. So we obtain weights of the beamforming and the optimal power of relays. Moreover, we advise a reasonable number of the relays by numerical simulation. 


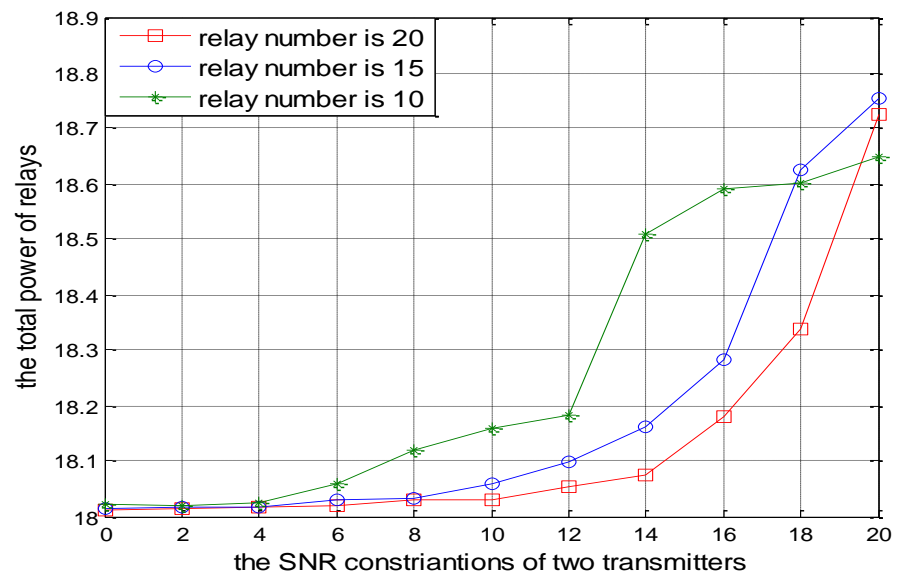

\section{Figure 2. $\mathrm{N}=10,15,20$, Transmit Power of the Relays vs SNRs of Two Transmitters}

\section{Acknowledgements}

This research is supported by National Natural Science Foundation of China under grant numbers 10971017 and 61375066.

\section{Appendix}

Lemma 1 Let $X$ be a positive semidefinite matrix of rank $r,{ }_{A}$ be a given symmetric matrix .Then, there is a decomposition of $X$

$$
X=\stackrel{̊}{j=1}_{j}^{r} x_{j} x_{j}^{T},
$$

Such that for all $j$,

$$
x_{j}^{T} A x_{j}=A ?\left(x_{j} x_{j}^{T}\right) \quad \frac{A \cdot X}{r}
$$

See reference [9].

Lemma 2 Suppose that $Z \hat{\mathrm{I}} H^{n}$ is a complex Hermitian positive semidefinite matrix of rank $r$ and $A, B \hat{\mathrm{I}} H^{n}$ be two given Hermitian matrices. Then, there is a rank-one decomposition of $Z$

$$
Z=\stackrel{a}{j=1}_{\AA^{r}} z_{j} z_{j}^{H},
$$

Such that

$$
z_{j}^{H} A z_{j}=\frac{A \cdot Z}{r}, z_{j}^{H} B z_{j}=\frac{B \cdot Z}{r}
$$

See references [10] and [11]. 


\section{References}

[1] J. Mitola III, "Cognitive radio: an integrated agent architecture for software defined radio", PhD Dissertation, KTH, Stockholm, Sweden, (2000) December.

[2] J. Mitola and G. Q. Maguire, "Cognitive Radios: Making Software Radios More Personal", IEEE Pers. Communications, vol. 6, no. 4, (1999) August, pp. 13-18.

[3] S. Haykin, "Cognitive Radio: Brain-Empowered Wireless Communications", IEEE J. Selected Areas Communications, vol. 23, no. 2, (2005) February, pp. 201-220.

[4] K. Jitvanichphaibool, Y.-C. Liang and R. Zhang, "Beamforming and Power Control for Multi-Antenna Cognitive Two-Way Relaying", WCNC, (2009).

[5] Y. Guo, G. Kang, N. Zhang, W. Zhou and P. Zhang, "Outage performance of relay assisted cognitive radio system under spectrum sharing constraints", IEE Electron. Lett., vol. 46, no. 2, (2010) January.

[6] Y. Zou, J. Zhu, B. Zheng and Y.-D. Yao, "An adaptive cooperation diversity scheme with best-relay selection in cognitive radio networks", IEEE Trans. Signal Process, vol. 58, no. 10, pp. 5438C5445, (2010) October.

[7] W. Su, F. Chen, D. A. Pados and J. D. Matyjas, "The outage probability and optimum power assignment for differential amplify-and-forward relaying", Proceedings of IEEE International Conference on Communications (ICC), Cape Town, South Africa, (2010) May, pp. 1-5.

[8] R. Mudumbai, D. R. Brown, U. Madhow and H. V. Poor, "Distributed transmit beamforming: Challenges and recent progress", IEEE Commun. Mag., vol. 47, no. 2, (2009) February, pp. 102-110.

[9] Y. Ye, "Linear Conic Programming", Department of Management Science and Engineering, Stanford University, (2004) December.

[10] W. Ai, Y. Huang and S. Zhang, "New results on Hermitian matrix rank-one decomposition", Mathematical Programming, Series A, vol. 128, (2011), pp. 253-283.

[11] Y. W. Huang and S. Zhang, "Complex matrix decomposition and quadratic programming", Mathematics of Operations Research, vol. 32, no. 3, (2007), pp. 758-768.

[12] T. Yi, L. Guo, K. Niu, H. Cai, J. Lin and W. Ai, "Cooperative beamforming in cognitive radio network with hybrid relay", Telecommunications (ICT), 19th International Conference, (2012).

[13] H. Cai, Y. Wang and T. Yi, "An approach for minimizing a quadratically constrained fractional quadratic problem with application to the communications over wireless channels", Optimization Methods and Software, vol. 29, no. 2, (2014), pp. 310-320.

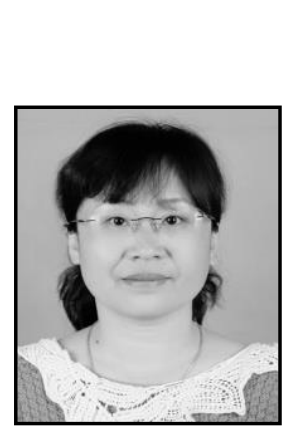

Author

Hongyan Cai received the M.Sc degree in applied mathematics from Beijing Normal university, Beijing, China in 2002. She is studying for a $\mathrm{Ph} . \mathrm{D}$ in optimization theory and methods in the School of Economics and Management, Beijing University of post and telecommunication, Beijing, China.

She is a teacher in Hebei University of Economics and business, Shijiazhuang, China. Her research interests include optimization theory and methods, cognitive radios, multiuser communication. 